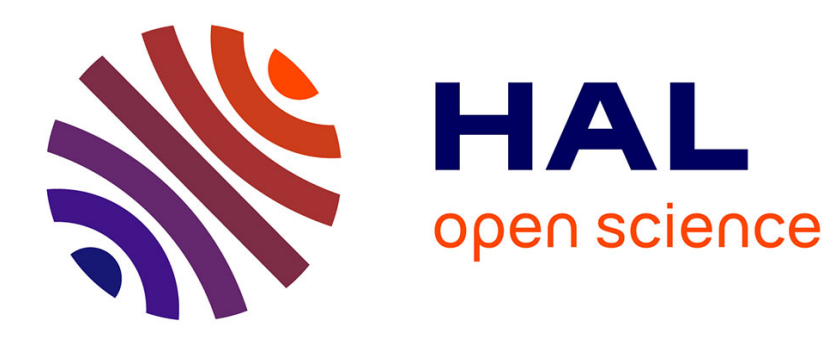

\title{
Application du retournement temporel au contrôle non destructif
}

\author{
N. Chakroun, M. Fink, F. Wu
}

\section{To cite this version:}

N. Chakroun, M. Fink, F. Wu. Application du retournement temporel au contrôle non destructif. Journal de Physique IV Proceedings, 1994, 04 (C5), pp.C5-1161-C5-1164. 10.1051/jp4:19945255 . jpa-00252944

\section{HAL Id: jpa-00252944 https://hal.science/jpa-00252944}

Submitted on 1 Jan 1994

HAL is a multi-disciplinary open access archive for the deposit and dissemination of scientific research documents, whether they are published or not. The documents may come from teaching and research institutions in France or abroad, or from public or private research centers.
L'archive ouverte pluridisciplinaire HAL, est destinée au dépôt et à la diffusion de documents scientifiques de niveau recherche, publiés ou non, émanant des établissements d'enseignement et de recherche français ou étrangers, des laboratoires publics ou privés. 


\title{
Application du retournement temporel au contrôle non destructif
}

\author{
N. CHAKROUN, M. FINK et F. WU
}

Laboratoire Ondes et Acoustique, ESPCI, 10 nue Vauquelin, 75005 Paris, France

\begin{abstract}
Résumé
L'utilisation d'un miroir ultrasonore à retournement temporel est une méthode originale pour détecter de petits défauts situés dans des solides hétérogènes de forme complexe. Le Retournement Temporel (RT) est une technique auto-adaptative qui réalise en temps réel une procédure de focalisation adaptée à la fois aux géométries de la sonde et de l'interface solideliquide, aux caractéristiques du milieu de propagation et à la forme du défaut. Dans les milieux solides très diffusants le niveau du bruit de speckle est élevé et il gêne la détection des défauts. Le RT permet d'améliorer cette détection, d'une part en focalisant de façon optimale sur les défauts et d'autre part en réduisant le niveau du bruit. En effet, cette technique se comporte différemment selon que les signaux proviennent d'un défaut ou du bruit de speckle. De plus, en itérant le processus de RT, il est possible de différencier lors d'un contrôle les zones contenant un défaut des zones ne contenant que du bruit de speckle.

\section{Abstract}

Ultrasonic Time Reversal Mirror (TRM) is an original solution to detect small cracks in solids of any geometrical shape. This technique is self adaptative. It achieves in real time, a natural focusing process matched to the defect shape, to the geometry of the liquid-solid interface and to the caracteristics of the propagation medium. In highly scattering media, the detection of small cracks is usually difficult due to the speckle noise. It is shown that TRM process allows a new approach of speckle noise reduction: TRM behaves differently if the signals come from an echographic speckle noise or from a defect. Using this property of TRM process, we developed an original signal processing that allows to distinguish, during the inspection, a high level speckle noise zone from a defect zone.
\end{abstract}

\section{Introduction}

Le speckle acoustique lié à la nature cohérente du rayonnement ultrasonore et à la nature diffusante des milieux solides hétérogènes masque les petits défauts et empêche leur détection par les méthodes conventionnelles utilisant un transducteur monoélément. Les Miroirs à Retournement Temporel (MRT) permettent d'améliorer la détection de ces défauts dans des structures bruitées. Cette technique autoadaptative réalise en temps réel une procédure de focalisation adaptée à la fois à la forme particulière du défaut, à la géométrie de l'interface solide-liquide, aux caractéristiques du milieu de propagation et à la géométrie de la sonde. Les distorsions du front d'onde introduites lors du trajet aller sont compensées lors du trajet retour.

Le miroir à retournement temporel est constitué d'un réseau de transducteurs ultrasonores émetteursrécepteurs à deux dimensions fonctionnant à 3 et $5 \mathrm{MHz}$. Chaque transducteur est relié à sa propre électronique. Ces transducteurs sont capables de mesurer une pression instantanée et d'émettre une grande variété de signaux.

La focalisation par RT en C.N.D. est obtenue en quatre étapes (Fig.1). Le miroir émet une onde brève vers le solide à inspecter. Si un réflecteur est présent dans le volume insonifié, il recevra une partie de 
l'énergie incidente et se comportera comme une source acoustique renvoyant un écho vers le réseau de transducteurs. Le front d'onde correspondant est capté par tous les éléments du miroir. Ce champ est échantillonné, numérisé, stocké, retourné temporellement et retransmis en même temps par tous les transducteurs vers le milieu. Cette procédure convertit une onde divergente issue d'un réflecteur en une onde convergente focalisée sur ce réflecteur. Cette focalisation de toute l'énergie du système sur le réflecteur amplifie considérablement sa signature acoustique et sa détection est rendue possible.

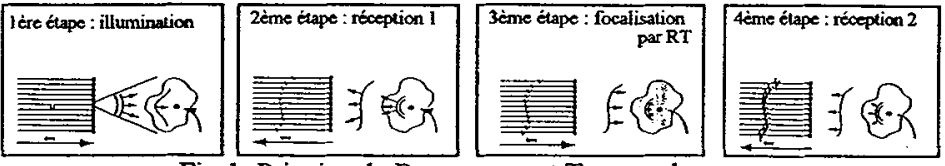

Fig. 1: Principe du Retournement Temporel

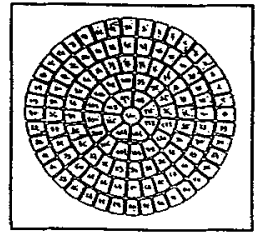

Fig. 2 : Miroir à RT

Un prototype de miroir à retournement temporel comprenant 128 voies et fonctionnant en temps réel a été construit. Il a permis de réaliser un grand nombre d'expériences sur des échantillons de duralumin de titane et d'acier, démontrant que le principe du RT est applicable au C.N.D.

Le RT améliore considérablement le rapport signal à bruit des échos des petits défauts dans les solides de structures bruitées, comparé aux méthodes monoélément conventionnelles. Cette amélioration est due à la différence de comportement du RT selon que les signaux proviennent d'une source cohérente (défaut) ou d'une source incohérente (microstructure diffusante). Deux approches du RT sont possibles. $\mathrm{Si}$ on n'effectue qu'une opération de RT toute l'énergie du système reconverge vers la source uniquement si elle est cohérente. Après deux RT il est possible de différencier les zones de bruit des zones contenant un défaut. Ce traitement très simple compare les fronts d'onde reçus par la sonde après une et deux itérations de RT.

\section{Retournement Temporel de signaux provenant d'une zone contenant un défaut.}

Les expériences ont été réalisées avec un réseau 2-D travaillant à $5 \mathrm{MHz}$, sur des pièces de titane présentant un niveau de bruit ultrasonore élevé. Ce miroir à retournement temporel composé de 121 éléments est un miroir sphérique de $330 \mathrm{~mm}$ de rayon de courbure (Fig.2). Le défaut est un TFP (trou à fond plat) de $0.4 \mathrm{~mm}$ de diamètre situé à $20 \mathrm{~mm}$ de l'interface eau/titane.

La première onde est émise par l'élément du centre vers la pièce de titane. Les échos provenant de la pièce sont enregistrés. La figure 3 transcrit l'amplitude des 121 signaux échographiques reçus en niveau de gris. Les signaux (enveloppes des signaux logarithmiques) sont présentés en mode B-Scan, où l'axe horizontal correspond au temps (profondeur) et l'axe vertical représente le numéro des transducteurs. On distingue aisément sur chacun des transducteurs les échos provenant des deux interfaces avant et arrière de la pièce de titane (Fig.3-a). Entre ces deux échos on observe le bruit de "speckle" renvoyés par les inhomogénéités du titane. L'écho du défaut n'est pas mis en évidence car il est "noyé" dans ce bruit de speckle. On sélectionne l'origine et la durée des signaux à retourner temporellement à l'aide d'une fenêtre temporelle identique pour tous les transducteurs. Une fenêtre de durée $2 \mu \mathrm{s}(6 \mathrm{~mm}$ de titane) est choisie autour de la profondeur du défaut (Fig. 3 -a). Ces signaux sont retournés temporellement et transmis vers le milieu. Sur les nouveaux signaux reçus de la pièce (Fig.3-b), on distingue toujours les échos d'interface et entre eux à la profondeur du défaut on voit très clairement une ligne courbe qui correspond à l'écho du défaut. Après un RT, la signature acoustique de ce défaut a été amplifiée car toute l'énergie acoustique du système a été refocalisée sur lui. La somme incohérente est une présentation plus compacte des 121 signaux reçus, la détection d'un défaut est matérialisée par l'apparition d'un pic localisé dans la fenêtre de retournement. Ce signal est le résultat de la somme des 121 enveloppes logarithmiques. La dynamique de ce système est de $90 \mathrm{~dB}$, on observe sur la somme incohérente qu'après un RT l'écho du défaut est à $30 \mathrm{~dB}$ au dessus du niveau du bruit (Fig.3-b). Cette expérience peut être reprise pour différentes positions du TFP dans le volume insonifié. Ce TFP est détecté sur une zone d'environ $1 \mathrm{~cm}^{2}$. 


\section{Retournement Temporel de signaux provenant d'une zone contenant du bruit de speckle.}

Après un RT dans une zone ne contenant que du bruit de speckle on n'observe aucun front d'onde à la position de la fenêtre de RT (Fig.4-b). Aucun signe de focalisation n'est observé. La somme incohérente ne présente pas de pic; ce qui signifie que les 121 signaux reçus sont complètement décorrélés. On note ici la différence de comportement du RT selon que les signaux proviennent d'un défaut ou du bruit de speckle. Dans les pièces de titane le bruit de speckle provient d'une microstructure dont les dimensions sont faibles devant la longueur d'onde $(\lambda / 100)$. Les détails de cette microstructure ne sont donc pas résolus à l'échelle de la longueur d'onde. Le RT ne peut pas focaliser l'énergie du système sur la source acoustique qui a donné naissance à ce bruit de speckle parce qu'il perd les informations sur les détails de la microstructure lors de la propagation inverse et directe du champ acoustique.

En itérant le processus il est possible d'améliorer encore les performances de RT. En comparant les fronts d'onde reçus par la sonde après un et deux RT il est ainsi possible de distinguer lors d'un contrôle l'origine des échos rétrodiffusés et de distinguer les zones de bruit des zones contenant un défaut.

\section{Le Retournement Temporel : une méthode efficace pour distinguer l'origine des échos.}

\section{4-1 Mode itératif du Retournement Temporel}

Lorsqu'on itère le processus de RT dans une zone contenant un défaut, on observe dans la fenêtre de RT deux fronts d'onde identiques sur les réceptions 2 et 3 (Fig. 3-b et 3-c). Pour un même transducteur, on présente les signaux reçus dans la fenêtre de RT après un et deux RT (Fig. 5). Ces deux signaux se ressemblent à un facteur d'amplification près : après un deuxième RT la focalisation de l'énergie sur ce défaut a été améliorée par le système.

$\mathrm{Si}$ on itère le RT dans une zone ne contenant que du bruit de speckle aucun front d'onde n'apparait sur les deux réceptions (Fig.4-b et 4-c). En observant sur le même transducteur que précédemment les signaux reçus après un et deux $\mathrm{RT}$, on s'aperçoit que les signaux sont décorrélés (il proviennent d'une source incohérente) et qu'il n'y a pas de phénomène de focalisation d'énergie (l'amplitude des deux signaux est identiques) (Fig. 6).

\section{4-2 Histogramme des écarts}

Nous avons développé un algorithme de corrélation simple et rapide pour évaluer le degré de ressemblance des fronts d'onde reçus après un et deux RT. La courbe donnant la position des maxima des signaux dans la fenêtre de RT est calculée pour chacune des réceptions 2 et 3 . Le nouveau coefficient de corrélation donne le taux de ressemblance de ces deux courbes. Pour l'élément $\mathrm{m}$, on note $\mathrm{T}_{\mathrm{m}}^{2}$ la position du maximum de l'amplitude du signal dans la fenêtre de $\mathrm{RT}$ pour la réception 2, et $\mathrm{T}_{\mathrm{m}}^{3}$ la position du maximum pour la réception 3 . On note $T_{m 1}$ la différence entre $T_{m}^{3}$ et $T_{m}^{2}$. Cette différence représente le déplacement du maximum de pression entre la réception 2 et la réception 3 . Cette opération est répétée pour les 121 éléments de la sonde. La dispersion de ces 121 valeurs de $T_{m}$ est présentée sous forme d'histogramme. Si les deux courbes de maxima se ressemblent (zone de défaut) (Fig 7-a et 7-b) l'histogramme des écarts présente un pic étroit dont le maximum est proche du nombre de transducteurs (Fig.7-c): les positions relatives des 121 maxima ne varient pas d'un RT au suivant. Par contre si les deux courbes de maxima sont décorrélés (zone de bruit) (Fig 8-a et 8-b) la valeur du maximum de l'histogramme est proche de zéro, et celui-ci est plat (Fig 8-c)

\section{Conclusion}

Le RT améliore des performances de détection de petits défauts dans les matériaux présentant un niveau élevé de bruit de speckle en augmentant le rapport signal à bruit. Le RT focalise de façon optimale toute l'énergie du système sur les défauts car les distorsions du front d'onde introduites lors du trajet direct sont compensées lors du trajet inverse. D'autre part, le RT réduit limportance du bruit de speckle car on ne peut 
pas refocaliser sur les détails de la microstructure qui ne sont pas résolus à l'échelle de la longueur d'onde. De plus au moyen de l'itération du RT on peut distinguer l'origine des échos grâce à l'histogramme des écarts. Actuellement il est possible de détecter un défaut de $0.4 \mathrm{~mm}$ de diamètre à $65 \mathrm{~mm}$ de profondeur dans des pièces de titane bruité.

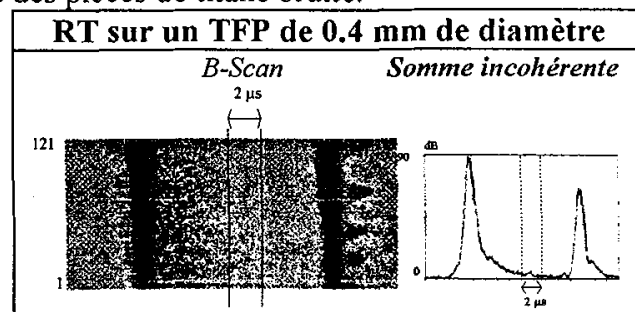

(a)

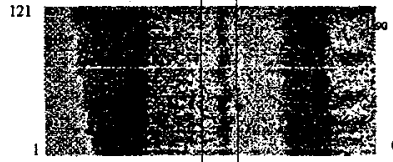

(b)

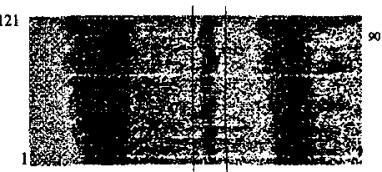

(c)

Fig. 3 : a) réception 1 (avant $\mathrm{RT}$ ), b) réception 2 (après un $\mathrm{RT}$ ), c) réception 3 (après deux $\mathrm{RT}$ )

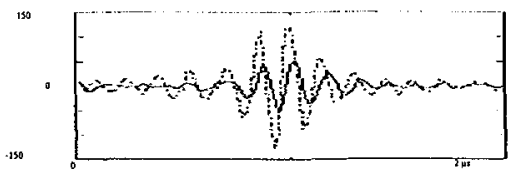

Fig 5 : Signaux reçus par le transducteur $n^{\circ} 20$ après un (ligne continue) et deux RT (ligne discontinue)
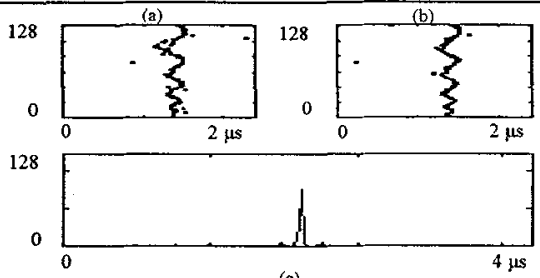

Fig. 7 : Courbe des maxima a) après un RT, b) après deux RT, c) Histogramme des écarts

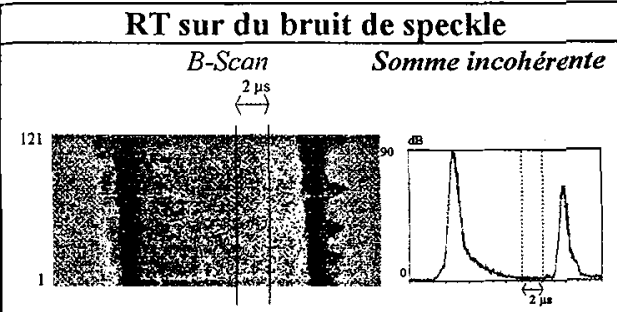

(a)

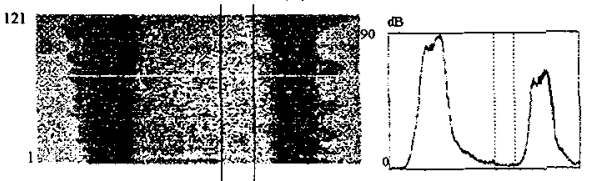

(b)

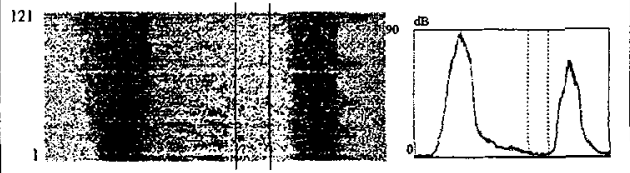

(c)

Fig. 4 : a) réception 1 (avant $\mathrm{RT}$ ), b) réception 2 (après un $\mathrm{RT}$ ), c) réception 3 (après deux $\mathrm{RT}$ )

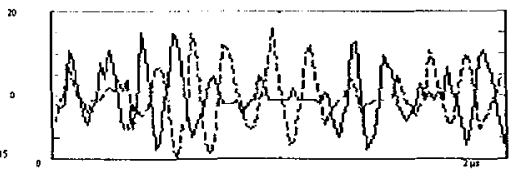

Fig.6: Signaux reçus par le transducteur $n^{\circ} 20$ après un (ligne continue) et deux RT (ligne discontinue)
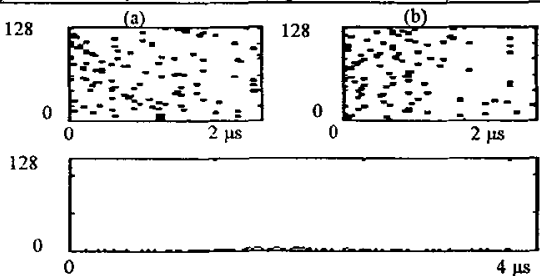

(c)

Fig.8 : Courbe des maxima a) après un $R T$, b) après deux RT, c) Histogramme des écarts

\section{Références}

[1] M. Fink, C. Prada, F. Wu, and D. Cassereau, "Self focusing with time reversal mirror in inhomogeneous media," Proc IEEE Ultrason. Symp. 1989, Montreal, PQ, Canada, vol.2, 1989, pp.681-686.

[2] C. Prada, F. Wu, and M. Fink, "The iterative time reversal mirror : A solution to self focusing in pulse-echo mode," J. Acoust. Soc. Amer., July 1991.

[3] M. Fink, "Time reversal of ultrasonic fields - Part I : Basic principles," IEEE Trans. Ultrason., Ferroelec., Freq. Contr., vol. 39, $\mathrm{n}^{\circ} 5$, September 1992.

[4] F. Wu, J.L Thomas, and M. Fink, "Time reversal of ultrasonic fields - Part II : Experimental results," IEEE Trans. Ultrason., Ferroelec., Freq. Contr., vol. 39, n5, September 1992.

[5] N. Chakroun, M. Fink, F. Wu, "Ultrasonic non destructive testing with Time Reversal Mirrors," Proc IEEE Ultrason. Symp. 1992, Tucson, U.S.A, vol.2, 1992, pp809-814. 